\title{
Expression of histo-blood group antigens in bronchial squamous metaplasia
}

\author{
N. Sánchez-Mora*\#, M. Cebollero Presmanes*, V. Monroy ", \\ M. Herranz Aladro* and E. Álvarez-Fernández*
}

ABSTRACT: The aim of the present study was to evaluate the expression of blood group antigens in squamous bronchial metaplasia in order to determine whether this factor could identify patients at risk of lung cancer.

In total, 100 bronchial biopsies were included in the present study. The cases were classified according to the World Health Organization grading system. Immunohistochemical stains for histo-blood groups A and B, and reactivity tests to p53 and the cellular proliferation index were performed.

A total of $56(56 \%)$ patients belonged to blood group A. Among them, six (10.7\%) patients who did not express antigen in squamous metaplasia, showed carcinoma at the moment of the biopsy $(n=3)$ or developed synchronous lung carcinoma $(n=3)$. A total of nine $(9 \%)$ patients belonged to blood group B. Loss of antigenic expression was observed in five cases. All of them developed synchronous lung carcinoma. The patients with low- and high-grade dysplasia developed lung cancer in 71 and $100 \%$ of the cases, respectively.

In conclusion, the findings of the present study suggest that the loss of histo-blood antigens expression is an event in the carcinogenesis of bronchial mucosa and it is usually associated with high-grade lesions and hyperproliferative activity.

KEYWORDS: Histo-blood group, squamous dysplasia, squamous metaplasia

$\mathbf{L}$ ung carcinogenesis is a multiple-step process characterised by accumulation of successive molecular genetic and epigenetic abnormalities, resulting in epithelial cell malignant transformations [1, 2], such as expression of oncogenes, and loss of tumour-supressor genes [2, 3]. Increasing information is currently available on the genetic alterations leading to the final invasive stage of bronchial carcinomas. However, the natural history of the pre-invasive lesions is poorly understood. Currently, it is clear that high-grade pre-invasive lesions will develop into invasive carcinoma in $30-50 \%$ of the cases, whereas the majority of low-grade pre-invasive lesions remain stable or regress during follow-up $[4,5]$. Nevertheless, increased efforts to better characterise lesions are needed.

Histo-blood group antigens are a group of glycoproteins and glycolipids whose antigenic specificity is determined by a variation in their constituent carbohydrate chains [6]. These antigens are involved in various biological processes, such as cellular differentiation, maturation, proliferation, malignant transformation and intercellular signalling [7]. The prognostic significance of a loss of or a modified histo-blood group antigen
$(\mathrm{ABH})$ expression has been suggested in carcinomas of various sites, including the urinary bladder [8], the gastrointestinal tract [9] and the lungs [10-12]. DAVIDSOHN and Ni [10] demonstrated a loss of $\mathrm{ABH}$ determinants in pulmonary carcinomas. Subsequently, LEE et al. [11] and MolDVAY et al. [13] demonstrated that a reduction or deletion of antigenic expression correlated with a reduced survival rate. MATSUMOTO et al. [14] concluded that this loss of $\mathrm{ABH}$ blood group antigens in lung carcinomas correlated with their metastatic potential, especially with recurrence and haematogenous metastasis. Similar findings were reported by ICHIKAWA et al. [15].

The aim of the present study was to explore the significance of the expression of histo-blood antigens in squamous metaplasia and its association to other prognostic factors, such as oncoprotein p53 expression and cellular proliferation index (Ki67) according to immunohistochemical techniques.

\section{MATERIAL AND METHODS}

The study material was obtained from the files of the Pathology Dept of the Gregorio Marañon Hospital (Madrid, Spain). It consisted of bronchial
AFFILIATIONS

*Dept of Pathological Anatomy,

Gregorio Marañón General University Hospital, Madrid, Spain.

\#LABIEMET, Los Andes University, Venezuela, and

"Dept of Pathological Anatomy, La Raza National Medical Centre. Mexico.

CORRESPONDENCE

N. Sánchez-Mora

Hospital General Universitario

Gregorio Marañón

C/ Dr. Esquerdo No. 46

Dpto. de Anatomía Patológica

CP. 28007 Madrid

Spain

Fax: 34915868162

E-mail: histonora@yahoo.es

Received:

January 232006

Accepted after revision:

September 142006

STATEMENT OF INTEREST

None declared.

European Respiratory Journal

Print ISSN 0903-1936

Online ISSN 1399-3003 
biopsy specimens obtained during bronchoscopy performed between 1996-2000 from 100 patients with respiratory symptoms and clinical suspicion of lung cancer. The lesions were classified and graded according to the World Health Organization (WHO) classification [16].

Demographic information was obtained from the clinical records. Smokers were defined as patients who smoked at the time of biopsy. Ex-smokers were defined as those who had given up smoking $>1 \mathrm{yr}$ before the time of biopsy. Nonsmokers were defined as those patients who never acquired the habit. Tobacco consumption was evaluated as a pack-yr index. In the follow-up, synchronous carcinoma was defined as a carcinoma diagnosed within 1 yr after a biopsy, demonstrating squamous metaplasia. Metachronous carcinoma was defined as occurring $\geqslant 1$ yr after the biopsy.

\section{Preparation of tissues}

Tissues were fixed in a neutral buffered solution of $10 \%$ formalin, embedded in paraffin, sectioned at a thickness of $4 \mu \mathrm{m}$ and stained with haematoxylin and eosin.

\section{Immunohistochemical studies}

Sections 4- $\mu \mathrm{m}$ thick from paraffin-embedded biopsy specimens were cut and mounted on glass slides. The specimens were deparaffinised in xylene and rehydrated step by step with descending concentrations of ethanol. The sections were incubated at $37^{\circ} \mathrm{C}$ with $0.3 \% \mathrm{H}_{2} \mathrm{O}_{2}$ in absolute methanol for $10 \mathrm{~min}$, to block endogenous peroxidase. After being washed for 20 min with PBS of $\mathrm{pH} 7.2$, the specimens were incubated with the primary antibodies for $45 \mathrm{~min}$ in a moist chamber at room temperature (RT). The primary antibodies used were: anti-blood group A (Clon: T36; Signet Laboratories, Dedham, MA, USA; 1:60 dilution), anti-blood group B (Clon: CLCP-19B; Signet Laboratories; 1:60 dilution), anti-p53 (Clon: DO7; Novocastra, Newcastle, UK; 1:100 dilution) and the antibody against the Ki67 (Clon: MIB-1; DAKO Corporation, Carpinteria, CA, USA; 1:200 dilution). These were used after pre-treatment in a microwave oven $(15 \mathrm{~min}, 500 \mathrm{~W})$ in citrate buffer (10 mM, pH 6.0).

The sections were subsequently incubated with biotinylated anti-mouse and anti-rabbit immunoglobulin $\mathrm{G}$ and ligandbinding assay (DAKO Corporation) for $25 \mathrm{~min}$ at RT, then rinsed in PBS for $5 \mathrm{~min}$ and immersed in avidin peroxidase complex for $25 \mathrm{~min}$. Finally, the peroxidase was localised by treatment of the samples with a fresh mixture of diaminobenzidine and substrate for $10 \mathrm{~min}$. After washing in distilled water, the sections were lightly counterstained with haematoxylin, dehydrated in ethanol, cleared in xylene and coverslipped using permount.

Immunostaining to $\mathrm{A}$ and $\mathrm{B}$ anti-groups was defined as positive when the cells showed a granular staining in the cytoplasm and/or the cytoplasmic membrane, regardless of its intensity. Inmunohistochemical reactivity to p53 and Ki67 was recorded as the percentage of stained nuclei. The test was deemed positive when $>10 \%$ of the nuclei were stained. The intensity was divided into five groups according to the percentage of stained cells as follows: $0=0-10 \%,+=10-25 \%$, $++=26-50 \%,+++=51-75 \%$ and $++++=76-100 \%$.

\section{Statistical analysis}

The p-values were calculated using Chi-squared according to Yates' test and finally corrected using the exact Fisher's test when values were less than five. The association of variable strength, as well as the possible risk to develop any respiratory neoplasia, was performed using odds ratios and 95\% confidence intervals.

\section{RESULTS}

The characteristics of the 100 patients included in the present study are shown in table 1 . Bronchial lesions were graded according to the WHO grading system. One case showing angiogenic squamous dysplasia (ASD) was classified as presenting severe dysplasia. All the patients had respiratory symptoms related with lung cancer, but not yet lung cancer diagnosis.

A total of 56 (56\%) patients belonged to blood group A. All of them showed a positive staining for histo-blood group in endothelium and red blood cells in the biopsy sample. Among them, six (10.7\%) patients showed no expression of histo-blood A antigen in bronchial epithelium. One case (16.6\%) showed mild dysplasia, two cases (33.3\%) showed severe dysplasia, two cases showed in situ carcinoma (CIS; 33.3\%) and one case $(16.6 \%)$ microinvasive carcinoma. Three $(50 \%)$ of the six patients showed carcinoma at the time of biopsy. The other three were diagnosed with lung carcinoma during the first year after biopsy (i.e. synchronous). All cases with loss of expression of the histo-blood group A showed positivity for Ki67 and p53, according to previously described criteria (see Immunohistochemical studies). High-intensity Ki67-stained cells were located in the basal and intermediate levels of metaplastic epithelium with a mean of $70 \%$ of stained cells. Similar results were observed for the p53 protein, which had a mean of $85 \%$ of stained cells. The intensity of staining was very strong. The loss of A antigen showed a significant relationship with Ki67 positive staining $(p=0.03$; table 2$)$. Among the

\begin{tabular}{lc} 
TABLE 1 & Characteristics of the patients with bronchial \\
lesions included in the present study & 100 \\
Subjects & 92 \\
Males & $65.5(32-85)$ \\
Age yrs & \\
Smoking history & 19 \\
Current smokers & 73 \\
Ex-smokers & 8 \\
Never-smokers & $44-110)$ \\
Pack-yrs smoked & \\
Bronchial lesion & 4 \\
BCH & 42 \\
Metaplasia without dysplasia & 23 \\
Mild dysplasia & 17 \\
Moderate dysplasia & 6 \\
Severe dysplasia & 6 \\
ClS & 2 \\
Microinvasive carcinoma & \\
\hline & \\
Data are presented as n or mean (range). BCH: basal cell byperplasia; CIS: \\
carcinoma in situ.
\end{tabular}




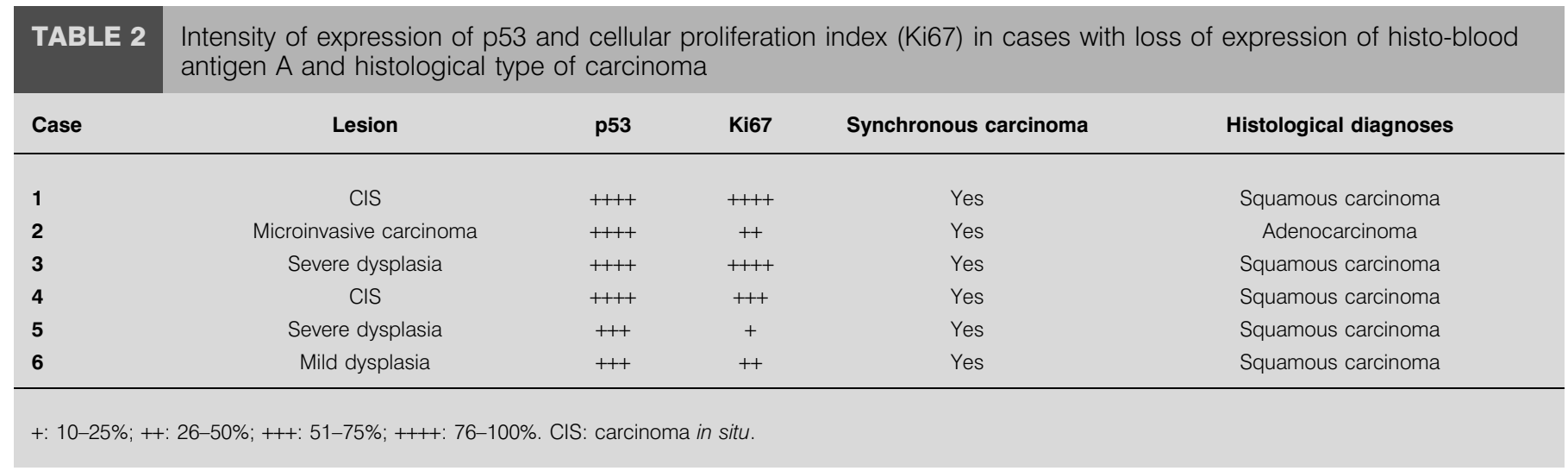

remaining cases without loss of expression of histo-blood group A, $22(39.3 \%)$ patients developed synchronous lung carcinoma, (12.5\%) seven cases developed metachronous lung carcinoma, 10 cases $(17.9 \%)$ did not develop neoplasia and 11 (19.6\%) cases were lost to follow-up. The case with ASD was associated with loss of histo-blood group A antigen expression, showed a positive stain to oncoprotein p53 and Ki67 and developed synchronous lung carcinoma (fig. 1).

Nine patients belonged to blood group B. Loss of antigenic expression was observed in five $(55 \%)$ cases, of which four showed moderate dysplasia and one case, mild dysplasia. All five $(100 \%)$ cases developed synchronous lung carcinoma. Positive staining of p53 and Ki67 was observed in all cases (table 3). Concerning the four remaining cases without loss of expression of histo-blood group B (45\%), three (33.3\%) cases developed metachronous lung carcinoma and one $(11.1 \%)$ case was lost to follow-up.

The predictive values of the loss of expression of histo-blood groups A and B antigens in pre-invasive bronchial lesions for the development of synchronouos and metachronous lung carcinoma were: a positive predictive value of $24 \%$ and a negative predictive value of $100 \%$.

Follow-up was available for 82 cases. The mean (range) followup period was $24.7(6-48)$ months. A total of $56(68.3 \%)$ cases developed synchronous carcinoma. Squamous lung cancer was the most frequent histological type in $28 \%$ of the cases. Small cell lung carcinoma developed in $6.1 \%$ of the cases, lung adenocarcinoma developed in $3.7 \%$ of the cases and $8.5 \%$ of the cases disclosed carcinoma in other localisations (six $(7.3 \%)$ cases laryngeal carcinoma and one $(1.2 \%)$ case breast carcinoma). Approximately $12.2 \%$ of the cases developed metachronous carcinomas; six squamous lung carcinoma, one case lung adenocarcinoma and three cases with carcinoma in other localisations (two cases in the larynx and one ureteral). In total, $18(21.9 \%)$ patients with clinical and radiological criteria of lung cancer, died without histological diagnoses. There was no development of neoplastic lesions in $16(19.5 \%)$ cases in the 48 months of follow-up (table 4).

After correlating the grade of dysplasia with the development of lung cancer in patients with follow-up, it was found that from the group of 55 patients bearing low-grade lesions (basal cell hyperplasia, metaplasia without dysplasia and mild dysplasia), 34 (61.8\%) patients developed synchronous carcinoma, five $(9.1 \%)$ patients developed metachronous carcinoma and $16(29.1 \%)$ patients did not develop cancer. From the group of 27 patients showing high-grade lesions (moderate and severe dysplasia), a total of 22 (81.5\%) developed synchronous carcinoma and five $(18.5 \%)$ developed metachronous carcinoma, demonstrating a statistically significant association between high-grade lesions and the development of lung cancer $(p=0.006)$. A total of $63(63 \%)$ cases showed staining of $>10 \%$ of the cell population of the plaque for Ki67 and $66(66 \%)$ cases for p53. When comparing Ki67 positivity and p53 with the grade of dysplasia, it becomes apparent that high-grade lesions show a higher number of positive cases than low-grade lesions ( 47.8 versus $77.4 \%, \mathrm{p}=0.005 ; 60.8$ versus $80.8 \%, \mathrm{p}=0.05$, respectively). a)

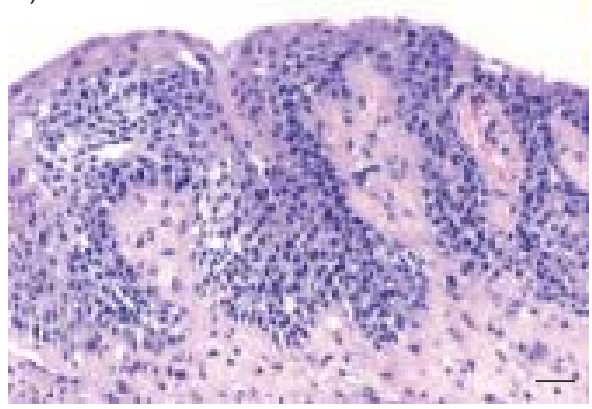

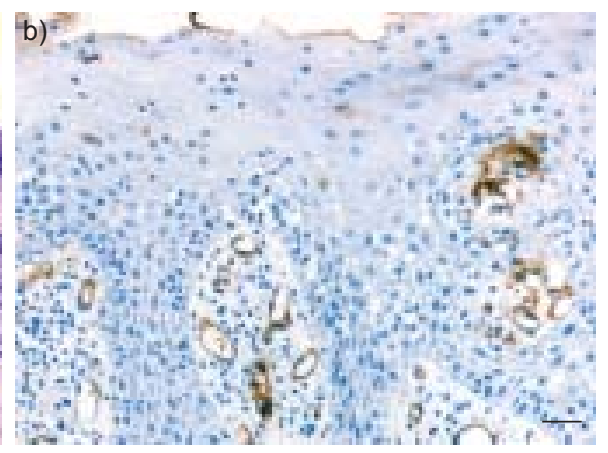

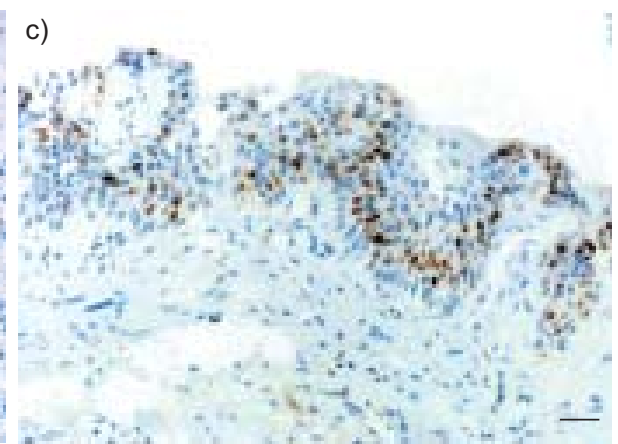

FIGURE 1. a) Angiogenic squamous dysplasia with haematoxylin and eosin staining. b) Positive immunohistochemical staining for histo-blood group $A$ in endothelium and negative in epithelium. c) Expression for p53 (immunohistochemical staining). a, b) Scale bars $=40 \mu \mathrm{m}$. c) Scale bar $=30 \mu \mathrm{m}$. 
TABLE 3 Intensity of expression of p53 and cellular proliferation index (Ki67) in cases with loss of expression of histo-blood antigen B and histological type of carcinoma

\begin{tabular}{lccccc} 
Case & Lesion & p53 & Ki67 & Synchronous carcinoma & Histological diagnoses \\
\hline $\mathbf{1}$ & Moderate dysplasia & + & ++ & Yes & Adenocarcinoma \\
$\mathbf{2}$ & Moderate dysplasia & + & ++ & Yes & Squamous carcinoma \\
$\mathbf{3}$ & Moderate dysplasia & +++ & +++ & Yes & Squamous carcinoma \\
$\mathbf{4}$ & Moderate dysplasia & +++ & + & Yes & Without histological diagnoses \\
$\mathbf{5}$ & Mild dysplasia & + & + & Yes & Without histological diagnoses \\
\hline
\end{tabular}

+: 1-25\%; ++: 26-50\%; +++: 51-75\%; ++++: 76-100\%.

\section{DISCUSSION}

The histological grading of squamous dysplastic changes is still used as a gold standard to address the malignant potential of those lesions [17]. However, a recent study by BREUER et al. [5] showed that $9 \%$ of the squamous metaplasias and low-grade (mild and moderate) dysplasias progressed to CIS and invasive carcinoma. This suggests that a step-wise histopathologic multistage development of lung carcinoma does not always occur or it is not always detected due to its rapid progression. BREUER et al. [5] concluded that the histological grade of any pre-neoplastic lesion cannot be reliably used for accurate risk assessment of field carcinogenesis, as it is not possible to differentiate the true malignant potential of pre-neoplastic squamous lesions.

Although the risk of lung cancer increases with the presence of pre-invasive lesions, the molecular determinants predicting the irreversible progression to lung cancer have not yet been identified.

The demonstration of variations in the immunohistochemical profile of expression of histo-blood groups A and B antigens, similar to those seen in carcinoma, would be of clinical significance not only to prognosticate lung carcinomas but also as a useful tool for early diagnosis. Deletion or reduction of histo-blood A or B epitopes has been extensively studied in human cancer. After an initial report in gastric cancer [18], this phenomenon was also described and correlated with histological grade and metastatic potential in other gastrointestinal [9], lung [10-12], cervical [19], oral [20] and bladder carcinomas [8]. As far as bronchial carcinoma is concerned,

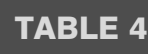

Histological types of carcinomas synchronous and metachronous developed by patients in the present study

Histological type of carcinoma Synchronous Metachronous Total

\begin{tabular}{lccc}
\hline Squamous lung carcinoma & 23 & 6 & 29 \\
Adenocarcinoma lung & 3 & 1 & 4 \\
Oat cell lung carcinoma & 5 & 0 & 5 \\
Carcinoma (other localisations) & & & \\
$\quad$ Squamous carcinoma of the larynx & 6 & 2 & 8 \\
$\quad$ Breast carcinoma & 1 & 1 & 1 \\
$\quad$ Ureteral carcinoma & 18 & 0 & 1 \\
$\quad$ Without histological diagnoses & 56 & 10 & 66 \\
Total & & & \\
\hline
\end{tabular}

previous reports have suggested that patients belonging to blood groups A or B and bearing tumours that do not express the corresponding histo-blood groups, show a shorter survival time after diagnosis [13, 14, 21]. Regarding histo-blood antigen expression, the present results suggest that antigenic loss occurs in the cancerisation process of bronchial mucosa, as it is a rare event in low-grade squamous lesions but a frequent one in highgrade lesions associated with developed synchronous lung carcinoma $[10-12,14,15]$. The present authors considered that, in spite of the fact that the size of the present series is too short to discuss predictive or diagnostic value, the trends found suggest that the loss of histo-blood antigen expression in pre-invasive bronchial lesions could reveal a severe field cancerisation process of bronchial mucosa and this can be a feature shown by pre-invasive bronchial lesions associated with synchronous carcinoma. It must be emphasised that the present study included patients with clinical suspicion of lung cancer.

The data analysed in the present study showed a high percentage $(68.3 \%)$ of synchronous cancer associated with pre-invasive bronchial lesions. This fact can be explained by two different hypotheses. First, it could support the theory that the sequential step-wise histological progression of preinvasive bronchial lesions does not always occur [5]. Secondly, it may reflect the fact that field cancerisation may randomly affect any site in the bronchial tree and, therefore, concomitant lesions can be of different age and progress toward invasion at different rates [22]. Unfortunately, the results of the present study cannot clarify this point.

The alteration of tumour suppressor genes plays a critical role in the development of many forms of cancer. The present authors have tried to correlate this factor with histo-blood antigen expression. The disturbance of the normal function of the p53 oncoprotein has been suggested to reflect an important event during malignant transformation. Some authors have reported data showing p53 overexpression in squamous bronchial metaplasia [23] and p53 mutations associated to high-grade dysplasia [1]. Other authors have studied metaplastic lesions from patients with coexisting carcinomas, showing p53 overexpression [23,24]. The results of the present study are in accordance with these data.

All cases with loss of expression of the histo-blood antigen also show a high staining to Ki67 and p53 in the present series. These results confirm that the loss of antigenic expression and other factors, such as p53 expression and increased cellular proliferation, are at least simultaneous events, rendering a 
larger series of cases to evaluate its statistical significance necessary. These findings support the progressive and stepwise accumulation of genetic/epigenetic abnormalities leading to cell transformation $[1,2,25,26]$.

ASD is characterised by small capillaries growing underneath the epithelium in a micropapillary pattern, indicating abnormal vascularisation of the bronchial mucosa. This is reflected by both increased microvascular density and aberrant morphology of the bronchial capillary bed. Possible mechanisms of angiogenesis in ASD may involve small populations of dysplastic squamous cells harbouring pre-malignant mutations transmitting angiogenic signals over very short distances [27]. In the present study only one case with ASD was observed. It was associated with loss of histo-blood group A antigen expression, oncoprotein p53 and Ki67 positivity and developed synchronous carcinoma. This finding gives additional support to the opinion that it must be classified as a high-grade lesion.

In conclusion, the present findings suggest that the loss of histoblood antigen expression is an event in the carcinogenesis of bronchial mucosa and is usually associated with high-grade lesions and hyperproliferative activity. Further studies are needed to better characterise the alterations that define the malignancy of the bronchial epithelium, as well as to identify critical factors, or association of factors, that would be predictive of the progression of pre-invasive bronchial lesions to invasive cancers. Together with very sensitive and specific methods and acceptable reproducibility to endobronchial detection of the malignant cell clones, such studies may help develop a more effective strategy for the management of this highly fatal disease.

\section{REFERENCES}

1 Jeanmart M, Lantuejoul S, Fievet F, et al. Value of immunohistochemical markers in preinvasive bronchial lesions in risk assessment of lung cancer. Clin Cancer Res 2003; 9: 2195-2203.

2 Hirsch FR, Merrick DT, Franklin WA. Role of biomarkers for early detection of lung cancer and chemoprevention. Eur Respir J 2002; 19: 1151-1158.

3 Thiberville L, Payne P, Vielkinds J, et al. Evidence of cumulative gene losses with progression of premalignant epithelial lesions to carcinoma of the bronchus. Cancer Res 1995; 55: 5133-5139.

4 Risse EK, Vooijs GP, van't Hof MA. Diagnostic significance of "severe dysplasia" in sputum cytology. Acta Cytol 1988; 32: 629-634.

5 Breuer RH, Pasic A, Smit EF, et al. The natural course of preneoplastic lesions in bronchial epithelium. Clin Cancer Res 2005; 11: 537-543.

6 Watkins WM. Biochemistry and genetics of the ABO, Lewis, and P blood group systems. Adv Hum Genet 1980; 10, 1-136: 379-385.

7 Sell S. Cancer-associated carbohydrates identified by monoclonal antibodies. Hum Pathol 1990; 21: 1003-1019.

8 Cuadrado E, Rodríguez-Trinidad A, Blasco E, Torrado J, Lopez García JA, Arozena F. Blood group isoantigens ABO $(\mathrm{H})$ in transitional carcinoma of the bladder: a clinicopathological study. J Urol 1986; 135: 409-415.

9 Slocombe GW, Berry CL, Swettenham KV. The variability of blood group antigens in gastric carcinoma as demonstrated by the immunoperoxidase technique. Virchows Arch A Pathol Anat Histol 1980; 387: 289-300.

10 Davidsohn I, Ni LY. Loss of isoantigens A, B, and H in carcinoma of the lung. Am J Pathol 1969; 57: 307-334.

11 Lee JS, Ro JY, Sahin AA, et al. Expression of blood-group antigen A--a favorable prognostic factor in non-small-cell lung cancer. N Engl J Med 1991; 324: 1084-1090.

12 Alvarez-Fernandez E, Carretero-Albinana L. Expression of blood group antigens by normal bronchopulmonary tissues and common forms of pulmonary carcinomas. Arch Pathol Lab Med 1991; 115: 42-49.

13 Moldvay J, Scheid P, Wild P, et al. Predictive survival markers in patients with surgically resected non-small cell lung carcinoma. Clin Cancer Res 2000; 6: 1125-1134.

14 Matsumoto $\mathrm{H}$, Muramatsu $\mathrm{H}$, Shimotakahara $\mathrm{T}$, et al. Correlation of expression of $\mathrm{ABH}$ blood group carbohydrate antigens with metastatic potential in human lung carcinomas. Cancer 1993; 72: 75-81.

15 Ichikawa D, Handa K, Withers DA, Hakomori S. Histoblood group A/B versus $\mathrm{H}$ status of human carcinoma cells as correlated with haptotactic cell motility: approach with A and B gene transfection. Cancer Res 1997; 57: 3092-3096.

16 Travis WD, Brambilla E, Müller-Hermelink HK, Harris CC, eds. Pathology and Genetics of Tumors of the Lung, Pleura, thymus and heart. WHO Classification of Tumor. Lyon, IARC Press, 2004.

17 Wistuba II. Histologic evaluation of bronchial squamous lesions: any role in lung cancer risk assessment? Clin Cancer Res 2005; 11: 1358-1360.

18 Oh-Uti K. Polysaccharides and a glycidamin in gastric cancer tissue. Tohoku J Exp Med 1949; 51: 297-304.

19 Davidsohn I, Stejskal R. Tissue antigens A, B and H in health and disease. Haematologia (Budap) 1972; 6: 177-184.

20 Dabelsteen E, Vedtofte P, Hakomori S, Young WW Jr. Accumulation of a blood group antigen precursor in oral premalignant lesions. Cancer Res 1983; 43: 1451-1454.

21 Graziano SL, Tatum AH, Gonchoroff NJ, Newman NB, Kohman LJ. Blood group antigen A and flow cytometric analysis in resected early-stage non-small cell lung cancer. Clin Cancer Res 1997; 3: 87-93.

22 Lam S, MacAulay C, Hung J, LeRiche J, Profio AE, Palcic B. Detection of dysplasia and carcinoma in situ with a lung imaging fluorescence endoscope device. J Thorac Cardiovasc Surg 1993; 105: 1035-1040.

23 Hirano T, Franzen B, Kato H, Ebihara Y, Auer G. Genesis of squamous cell lung carcinoma. Sequential changes of proliferation, DNA ploidy, and p53 expression. Am J Pathol 1994; 144: 296-302.

24 Sundaresan V, Ganly P, Hasleton P, et al. p53 and chromosome 3 abnormalities, characteristic of malignant lung tumours, are detectable in preinvasive lesions of the bronchus. Oncogene 1992; 7: 1989-1997.

25 Chyczewski L, Niklinski J, Chyczewska E, Niklinska W, Naumnik W. Morphological aspects of carcinogenesis in the lung. Lung Cancer 2001; 34: Suppl. 2, S17-S25.

26 Kerr KM. Morphology and genetic of pre-invasive pulmonary disease. Current Diagnostic Pathology 2004; 10: 259-268.

27 Keith RL, Miller YE, Gemmill RM, et al. Angiogenic squamous dysplasia in bronchi of individuals at high risk for lung cancer. Clin Cancer Res 2000; 6: 1616-1625. 